\title{
Four-Port Microstripline Antenna Integrated With a Distributed Amplifier
}

\author{
K. W. Eccleston* and S. Bommana \\ Dept of Electrical and Computer Engineering, \\ University of Canterbury, Christchurch, NEW ZEALAND \\ *Email: kim.eccleston@elec.canterbury.ac.nz
}

\begin{abstract}
In this paper, we propose a 4-port edge-fed rectangular microstripline antenna that is integrated with a 4-FET dual-fed distributed amplifier. The 4-port antenna is directly connected to the FETs and has the additional roles of power combining, harmonic suppression and FET biasing. This approach eliminates transmission lines and other elements that would otherwise be needed to realize the amplifier and connect it to an antenna, thereby saving circuit area and minimizing losses.
\end{abstract}

\section{INTRODUCTION}

Conventional microwave output stages may typically comprise power amplifier modules, a power combiner, harmonic filters, and an antenna that are separately designed and interconnected with transmission lines. These interconnecting transmission lines add extra losses that degrade overall performance. An alternative to is integrate these functions within one circuit [1]. In addition to eliminating the interconnections, the antenna and amplifier are designed to be mutually compatible with each other, and the antenna can have multiple roles such as harmonic tuning and suppression [1] [3], power combining [1][3]-[6], and dual mode [7].

The integrated antennas that have appeared in the literature have predominantly been edge-fed microstripline patches [3][6], or microstripline fed slot [2][3], though other geometries have also been proposed [1][7]. As far as integrated power combining is concerned, edge-fed microstripline patches [3]-[6] and microstripline fed slot [3] have been used, and in either case, due to their feeding and resonant behavior, harmonics can be suppressed [3].

$\mathrm{N}$-way combining requires an antenna with $\mathrm{N}$ ports. On the other hand, integrated antennas described in the literature that perform power combining [3] - [6] have been restricted to two ports. In this work we describe a 4-port edge-fed rectangular microstripline patch antenna which can be integrated with a dual-fed distributed amplifier [8].

\section{INTEGRATED 4-PORT MICROSTRIP ANTENNA}

The 2-port edge-fed microstripline patch antenna has been demonstrated as a feasible method of 2-way combining [3]-[6]. The ports are located at each end of a wide microstripline whose length is $\lambda / 2$ and these ports are driven anti-phase and this achieves even harmonic suppression in class-B push-pull amplifiers [3]. Moreover, the differential mode excitation radiates power [5][6].
This concept can be extended as shown in Fig. 1(a) to contain additional RF ports (ports 1 to 4 ) and a dc feed port. The RF ports are equally spaced and run down one edge of the patch to facilitate convenient connection to transistors. $\mathrm{L}$ is designed to be $3 \lambda / 2$ at the antenna centre frequency and hence the RF ports are spaced $\lambda / 2$ at the centre frequency. Since $L$ is $3 \lambda / 2$, the antenna will be resonant and when the RF ports ( 1 to 4) are fed with equal level but alternating relative phases of $0^{\circ}$ and $180^{\circ}$, the mode profile shown in Fig. 1(b) results. Such excitation can be achieved by driving the RF ports with the drains of FETs of a dual-fed distributed amplifier (DFDA) [8] as shown in Fig. 2. Or moreover, the patch forms the output transmission line of a DFDA [8]. For the above mentioned resonance mode, the electric field will be zero half-way between ports 2 and 3 and this means that a dc voltage source connected to port 5 (dc port) does not have a significant effect on the antenna radiation properties.

(a)

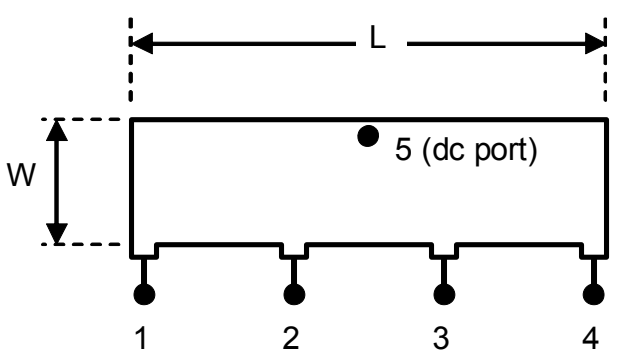

(b)

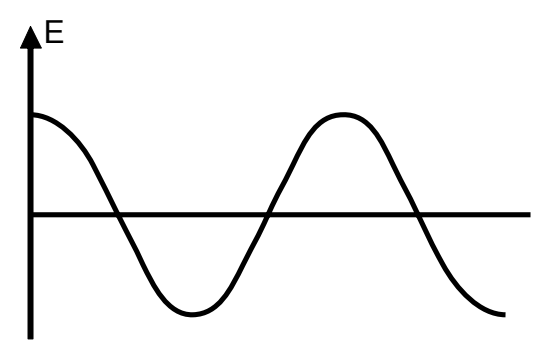

Fig. 1 Proposed four-port microstripline patch antenna: (a) outline, and (b) resonant mode profile.

It is important that the antenna RF port excitations have equal amplitude for the above mentioned mode to be faithfully excited. Since the drain currents of the FETs of a DFDA have identical amplitude (but relative phase alternating between $0^{\circ}$ and $180^{\circ}$ ), the antenna RF port impedances need to be identical 
at the centre frequency. This will also ensure that the FET loadlines are identical thereby sharing the burden of amplification equally among the FETs [8]. If the FET drain port is assumed to behave as an ideal current source [8] then it is appropriate to consider the antenna $\mathrm{Z}$-parameters $\mathrm{Z}_{\mathrm{j} j}(\mathrm{j}=1 . .4)$ with port 5 short circuited.

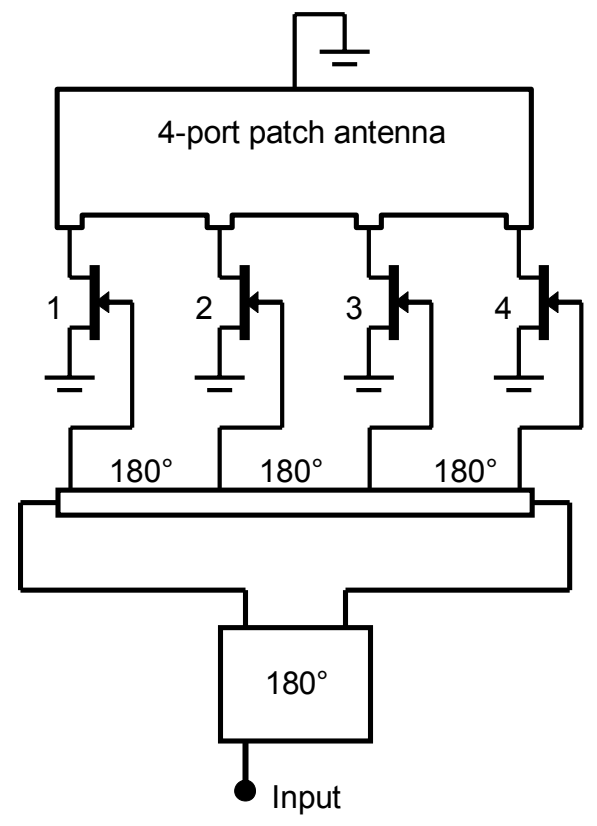

Fig. 2 RF schematic of proposed 4-port patch antenna integrated with a 4-FET DFDA.

The value of $\mathrm{W}$ can be adjusted to obtain a suitable radiation resistance at the centre frequency. However, if $\mathrm{W}$ is too large, transverse variation of patch surface current will be significant which results in reduced cross-polarisation isolation, and our investigations have also shown that the antenna RF port input impedances will increasingly differ. Since the radiation resistance of this type of antenna is high, step-impedance transformers are added at the ports of the antenna to present suitable loadlines for the FETs.

\section{ANTENNA DESIGN AND SIMULATION}

We considered the design of a 4-port antenna operating at $2.45 \mathrm{GHz}$ realized upon a substrate with thickness $0.787 \mathrm{~mm}$ and a dielectric constant of 2.2. Full-wave EM Simulations using Sonnet ${ }^{1}$ were used to aid the design of the antenna. During the simulations, port 5 was short-circuited, since a voltage source would be connected to this port. Due to the analysis method used by Sonnet, the side walls of the box are constrained to be metal. To minimize coupling between the antenna and its images in the side walls as well as ensure that sufficient number of modes can propagate toward the open-top, the box lateral dimensions were several wavelengths. To ensure that the fringing fields do not interact with the open-top boundary condition, the box height was around 0.5 wavelength.

\footnotetext{
${ }^{1}$ Sonnet is trademark of Sonnet Software Inc: www.sonnetusa.com
}

Given that the antenna is large and the above requirements, the grid spacing was set to $1 \mathrm{~mm}$ in both lateral directions to ensure reasonable computation time. This level of grid spacing also discretized the values of centre frequency that could be achieved. When $\mathrm{L}$ is $124 \mathrm{~mm}$ the centre frequency is 2.4628 $\mathrm{GHz}$ and $\mathrm{W}$ equal to $32 \mathrm{~mm}$ gave the best compromise between size, radiation resistance, and symmetry of port responses. Fig. 3 shows the frequency response of the $Z$-parameters $Z_{11}$ and $\mathrm{Z}_{22}$ with port 5 short-circuited; noting that by physical symmetry $Z_{33}=Z_{22}$ and $Z_{44}=Z_{11}$. The responses shown in Fig. 3 include the effects of the step-impedance transformers. It can be seen that $Z_{11}$ and $Z_{22}$ are nearly identical and that the $R e Z_{j j}$ is around $70 \Omega$ at the centre frequency.

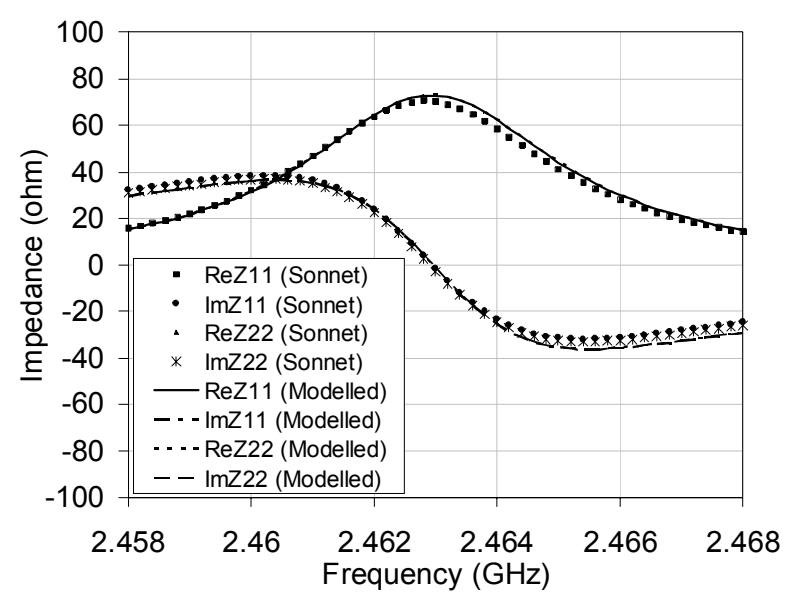

Fig. 3 Calculated response of $Z_{11}$ and $Z_{22}$ versus frequency for the antenna including step impedance transformers.

Fig. 4 shows the surface current density and radiation patterns at $2.4628 \mathrm{GHz}$ with ports equally excited but with alternating relative phases of $0^{\circ}$ and $180^{\circ}$. Fig. 4(a) demonstrates the purity of the mode of resonance. Fig. 4(b) shows an E-plane co-polar and cross-polar radiation patterns. Similarly Fig. 4(c) shows the H-plane radiation patterns. Simulations at other frequencies showed that the crosspolarisation isolation is greater than $20 \mathrm{~dB}$ over the bandwidth of the antenna. Further simulations determined that the DFDA gate line and input hybrid did not significantly affect the performance of the antenna.

\section{INTEGRATED ANTENNA AMPLIFIER SIMULATION}

The amplifier is an inherently nonlinear circuit so it is important for simulations that the antenna be characterized at both the fundamental and at harmonics. Due to the excessive computational times that would be required to analyse the antenna at harmonics of $2.4628 \mathrm{GHz}$ using Sonnet, a circuit model was used to represent the antenna during circuit simulations. This model is based upon the transmission line model [9]. The model parameters were fine-tuned so that the model agrees with Sonnet simulations for frequencies around the centre frequency. The model responses are depicted by the solid curves in Fig. 3. 
(a)

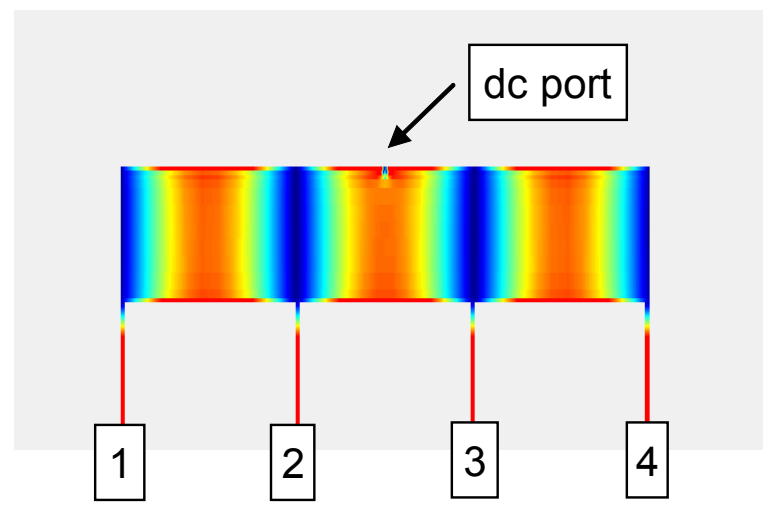

(b)

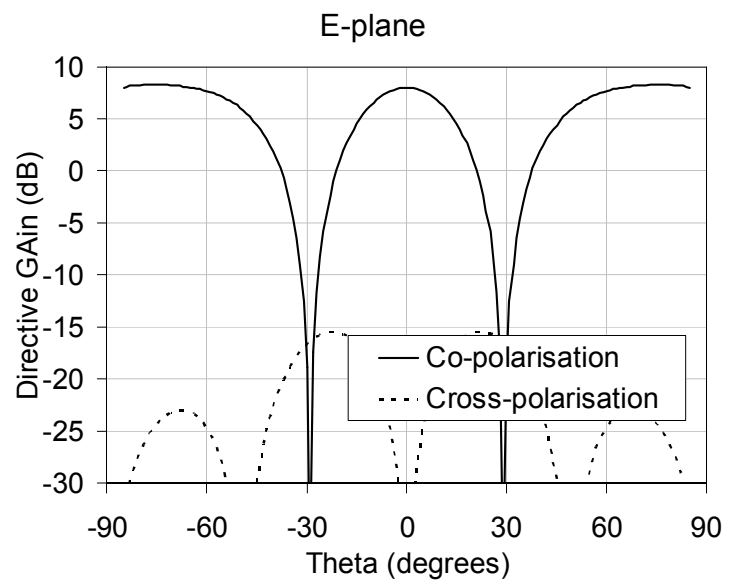

(c)

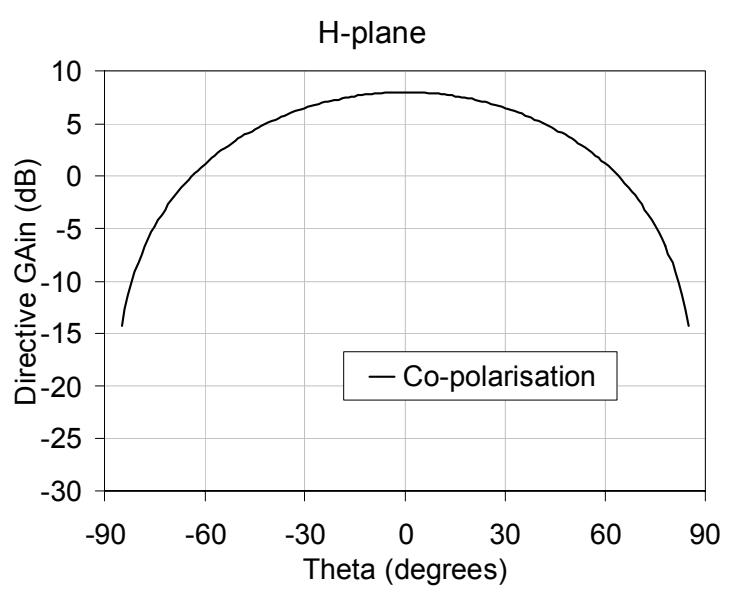

Fig. 4 Calculated response of the 4-port antenna at $2.4628 \mathrm{GHz}$ with ports equally excited but alternating $0^{\circ}$ and $180^{\circ}$ phase: (a) surface current density,

(b) E-plane radiation patterns, and (c) H-plane radiation pattern (crosspolarisation was negligible).

The FETs considered were FLK012WF / FLK017WF. These GaAs power FETs have a pinch-off voltage of $-2 \mathrm{~V}$ and a saturation drain current of $60 \mathrm{~mA}$ when $\mathrm{V}_{\mathrm{GS}}$ is zero. A Statz model [10] for the FET was used in the harmonic balance simulations. The drain supply voltage was $4 \mathrm{~V}$, and the gate voltage was $-1 \mathrm{~V}$ which established a quiescent drain current of $30 \mathrm{~mA}$ for each FET.

Fig. 5 shows the simulated drain current and drain voltage waveforms when the input generator available power was 8 $\mathrm{dBm}$ at $2.4628 \mathrm{GHz}$. It is clear that the drain voltage waveforms are nearly identical and current waveforms are similarly nearly identical (apart from the expected $180^{\circ}$ phase shift between ports) for each port. When the input power is increased, the drain voltage waveforms show the harmonic suppression capability of the antenna. The FET loads at this frequency are nearly pure real with a value of about $150 \Omega$, or in other words, $2 \mathrm{ReZ}_{\mathrm{ji}}$ at the centre frequency. This result is consistent with the DFDA principles [8].

(a)

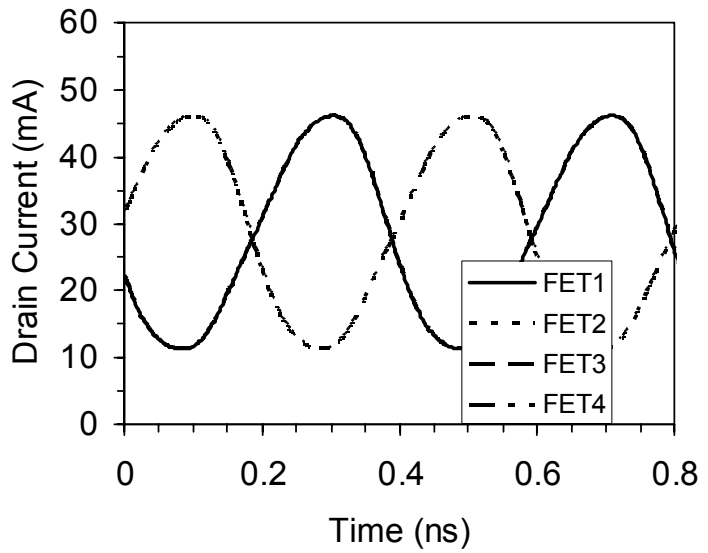

(b)

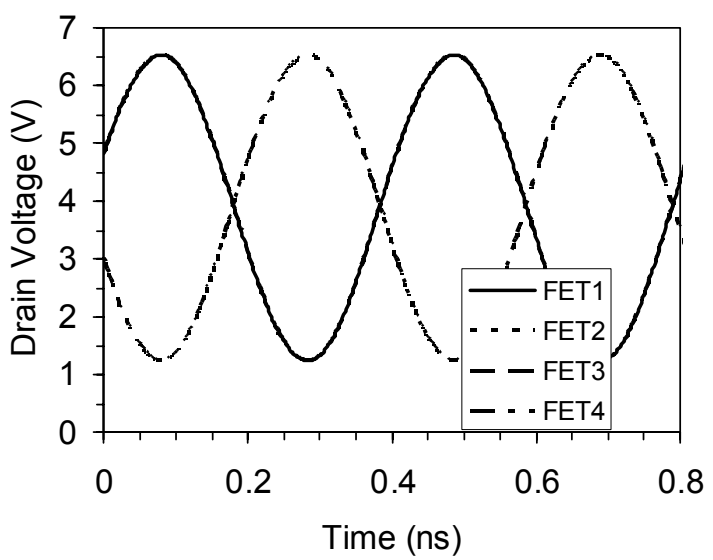

Fig. 5 Simulated FET and drain current and drain voltage waveforms. Input generator available power is $10 \mathrm{dBm}$ at a frequency of $2.4628 \mathrm{GHz}$.

\section{CONCLUSION}

In this paper we have described a multiport rectangular microstrip patch antenna that is designed for integration with a dual-fed distributed amplifier. The length of the patch is $3 \lambda / 2$, there are four RF ports equally spaced along one side that connect to the FET drains, and there is a dc port for biasing the drains of the FETs. Simulations of an integrated antenna designed for operation at $2.45 \mathrm{GHz}$ have demonstrated the 
feasibility of this approach. The antenna performs power combining, harmonic suppression, and FET biasing in addition to its principle role of radiator; thereby eliminating interconnects and other components that would otherwise be required.

\section{REFERENCES}

[1] V. Radisic, Y. Qian and T. Itoh, "Novel Architectures for High-Frequency Amplifiers for Wireless Applications", IEEE Trans. on Microwave Theory and Techniques, 46(11), pp 1901 - 1909, Nov. 1998.

[2] M. D. Weiss and Z. Popović, "A $10 \mathrm{GHz}$ High-Efficiency Active Antenna”, 1999 IEEE MTT-S International Microwave Symposium, pp 663 - 666.

[3] W. R. Deal, V. Radisic, Y. Qian and T. Itoh, "IntegratedAntenna Push-Pull Power Amplifiers", IEEE Trans. on Microwave Theory and Techniques, 47(8), pp 1418 - 1425, Aug 1999.

[4] W. Wang and Y. P. Zhang, "0.18- $\mu \mathrm{m}$ CMOS Push-Pull Power Amplifier With Antenna in IC Package", IEEE Microwave and Wireless Component Letters, 14(1), pp 13
- 15, Jan 2004.

[5] S. C. Gao, P Gardiner and S. T. Chiw, "Integrated Antenna for LINC Systems", Microwave and Optical Technology Letters, 33(2), pp 93 - 95, 20 April 2002.

[6] S. C. Gao and P. Gardiner, "Novel Integrated Antenna for LINC Power Amplifiers", 2002 IEEE Antennas and Propagation Symposium, pp 508 - 511.

[7] B. M. Lee, H. Kim and S. Kwon, "Dual-Feeding Active Integrated Antenna for Dual-Resonant Structure", Microwave and Optical Tech. Letters, 34(6), pp 435 - 437, 20 Sept 2000.

[8] K. W. Eccleston, "Design considerations for the dual-fed distributed power amplifier", 2000 Asia Pacific Microwave Conference (APMC 2000), 3 - 6 Dec 2000, Sydney, Australia, pp 205 - 208.

[9] A. G. Derneryd, "Linearly Polarized Microstrip Antennas", IEEE Trans. on Antenna and Propagation, Vol. 24, No. 6, pp 846 - 851, Nov. 1976.

[10] H. Statz, P. Newman, W. Smith, R. A. Pucel \& H. A. Haus, "GaAs FET device and circuit simulation in SPICE", IEEE Trans. on Electron Devices, 34 (2), pp 160-169, Feb. 1987. 